\title{
Quantum Dot Nanotechnology for Nano-Bio and Nano-Agri Applications
}

\author{
Swadeshmukul Santra \\ University of Central Florida \\ Orlando, FL 32816, USA \\ ssantra@ucf.edu
}

\begin{abstract}
Over the past two decades, Quantum dot (Qdot) nanotechnology has provided sophisticated tools for sensitive imaging of biological systems and sensing of biomolecules. In comparison to traditional organic dyes and fluorescent proteins, Qdots are much brighter and highly photostable. Real-time intracellular tracking of biological molecules and multiplexed imaging of intracellular organelles with high resolution have been possible using Qdot probes. In recent years, a number of Qdot based biosensing probes have been reported which could tremendously advance cancer drug discovery research by confirming release of therapeutic cargoes and possibly quantifying the released cargo amount within a single cell. In this talk, I will present an overview of our Qdot nanotechnology research program in my group including non-cytotoxic $\mathrm{Zn}$ based industry-grade Qdot as a delivery platform for small molecules such as antibiotics.
\end{abstract}

